\title{
AN INTRODUCTION TO ULRICH BUNDLES
}

\author{
ARNAUD BEAUVILLE
}

\begin{abstract}
After recalling the definition and basic properties of Ulrich bundles, we focus on the existence problem: does any smooth projective variety carry a Ulrich bundle? We show that the Serre construction provides a positive answer on certain surfaces and threefolds.
\end{abstract}

\section{INTRODUCTION}

Ulrich bundles have had three lives. They first appeared in the 80's in commutative algebra, starting with [U], under various names (linear maximal, or maximally generated, Cohen-Macaulay modules). They entered the realm of algebraic geometry twenty years later, with the beautiful paper [ES]. But it is only in the recent years that they have received wide attention among algebraic geometers.

In these notes we will first explain the definition and basic properties of Ulrich bundles, and motivate their study by the case of hypersurfaces. We will then focus on the fundamental and intriguing question of the subject, which goes back to [U]: does any smooth projective variety carry a Ulrich bundle? We will show that the classical Serre construction of rank 2 bundles give interesting examples for some surfaces and threefolds.

We will work over the complex numbers, though most results are valid over any algebraically closed field. We will denote the ambient projective space by $\mathbb{P}$ when we do not need to specify its dimension.

\section{ULRICH BUNDLE ON HYPERSURFACES}

We start with a classical problem: given a smooth hypersurface $X \subset \mathbb{P}$, can we write the equation of $X$ as a determinant of linear forms? The (positive) answer for a smooth cubic surface goes back at least to 1863 [S]. But determinantal hypersurfaces have singularities in codimension 3, so a smooth hypersurface of dimension $\geq 3$ cannot be written as a determinant.

Let us settle therefore for a weaker property: we ask whether $X$ can be defined set-theoretically by a linear determinant. This can be expressed nicely in terms of vector bundles on $X$ :

Proposition 1. Let $X$ be a smooth hypersurface of degree $d$ in $\mathbb{P}$, given by an equation $F=0$, and let $r$ be an integer $\geq 1$. The following conditions are equivalent:

1) $F^{r}=\operatorname{det}\left(L_{i j}\right)$, where $\left(L_{i j}\right)$ is a $r d \times r d$ matrix of linear forms on $\mathbb{P}$;

2) There exists a rank $r$ vector bundle $E$ on $X$ and an exact sequence $0 \rightarrow \mathscr{O}_{\mathbb{P}}(-1)^{r d} \stackrel{L}{\longrightarrow} \mathscr{O}_{\mathbb{P}}^{\text {rd }} \rightarrow E \rightarrow 0$.

Proof : If 2) holds, the determinant of $L$ vanishes exactly along $X$, hence is proportional to some power of $F$, thus to $F^{r}$ for degree reasons. 
Assume that 1) holds. We claim that the cokernel $E$ of the homomorphism $L: \mathscr{O}_{\mathbb{P}}(-1)^{r d} \rightarrow \mathscr{O}_{\mathbb{P}}^{r d}$ is a vector bundle on $X$. Let $x \in X$; the depth of the stalk $E_{x}$ as a $\mathscr{O}_{\mathbb{P}, x}$-module or as a $\mathscr{O}_{X, x}$-module is the same, and is equal by the Auslander-Buchsbaum formula to

$$
\operatorname{depth}\left(E_{x}\right)=\operatorname{dim}\left(\mathscr{O}_{\mathbb{P}, x}\right)-\operatorname{proj} \cdot \operatorname{dim}_{\mathscr{O}_{\mathbb{P}, x}}\left(E_{x}\right)=\operatorname{dim}\left(\mathscr{O}_{\mathbb{P}, x}\right)-1=\operatorname{dim}\left(\mathscr{O}_{X, x}\right),
$$

hence $E_{x}$ is a free $\mathscr{O}_{X, x}$-module, so $E$ is locally free on $X$.

The first Chern class of $E$ as a $\mathscr{O}_{\mathbb{P}}$-module is $-r d c_{1}\left(\mathscr{O}_{\mathbb{P}}(-1)\right)=r[X]$, hence $E$ has rank $r$.

The vector bundles $E$ which appear in 2) will be our main object of study. They can be characterized in a variety of ways:

Proposition 2. Let $X \subset \mathbb{P}$ be a smooth hypersurface of degree $d, E$ a rank $r$ vector bundle on $X$. The following conditions are equivalent:

1) There exists an exact sequence $0 \rightarrow \mathscr{O}_{\mathbb{P}}^{\text {rd }}(-1) \stackrel{L}{\longrightarrow} \mathscr{O}_{\mathbb{P}}^{\text {rd }} \rightarrow E \rightarrow 0$.

2) The cohomology $H \bullet(X, E(-p))$ vanishes for $1 \leq p \leq \operatorname{dim}(X)$.

3) If $\pi: X \rightarrow H$ is the projection from a point of $\mathbb{P} \backslash X$ to a hyperplane, the vector bundle $\pi_{*} E$ is trivial (hence isomorphic to $\mathscr{O}_{H}^{r d}$ ).

It turns out that this Proposition is a particular case of a more general result, which makes sense on any smooth projective variety:

Theorem 1 ([ES]). Let $X \subset \mathbb{P}$ be a smooth variety, and let $E$ be a vector bundle on $X$. The following conditions are equivalent:

1) There exists a linear resolution

$$
0 \rightarrow L_{c} \rightarrow L_{c-1} \rightarrow \ldots \rightarrow L_{0} \rightarrow E \rightarrow 0
$$

with $c=\operatorname{codim}(X, \mathbb{P})$ and $L_{i}=\mathscr{O}_{\mathbb{P}}(-i)^{b_{i}}$.

2) The cohomology $H_{\bullet}(X, E(-p))$ vanishes for $1 \leq p \leq \operatorname{dim}(X)$.

3) If $\pi: X \rightarrow \mathbb{P}^{\operatorname{dim}(X)}$ is a finite linear projection, the vector bundle $\pi_{*} E$ is trivial.

The vector bundle $E$ is a Ulrich bundle if it satisfies these equivalent conditions.

Proof : We first observe that the Theorem holds for $X=\mathbb{P}^{n}$; that is, a vector bundle $E$ on $\mathbb{P}^{n}$ with $H^{\bullet}(E(-p))=0$ for $1 \leq p \leq n$ is trivial. Indeed this implies $H^{p}\left(\mathbb{P}^{n}, E(-p)\right)=0$ for $p>0$, which means that $E$ is 0 -regular in the sense of Mumford [M] Lecture 14]; this implies in particular that $E$ is globally generated, and satisfies $H^{p}\left(\mathbb{P}^{n}, E\right)=0$ for $p>0$. The Hilbert polynomial $\chi(E(t))$ vanishes for $t=-1, \ldots,-n$, and its leading coefficient is $\frac{r}{n !}$; therefore $\chi(E(t))=\frac{r}{n !}(t+1) \ldots(t+n)$, hence $h^{0}(E)=r$. Thus we have a surjective homomorphism $\mathscr{O}_{\mathbb{P}^{n}}^{r} \rightarrow E$, which is necessarily an isomorphism.

Let us treat the general case. We put $n:=\operatorname{dim}(X)$. If 3) holds, we have $H \bullet(X, E(-i))=$ $H^{\bullet}\left(\mathbb{P}^{n}, \pi_{*} E(-i)\right)=0$ for $1 \leq i \leq n$, hence 2$)$. Conversely, if 2$)$ holds, the vector bundle $F:=\pi_{*} E$ on $\mathbb{P}^{n}$ satisfies $H^{\bullet}\left(\mathbb{P}^{n}, F(-i)\right)=0$ for $1 \leq i \leq n$, hence is trivial by the case already treated.

The implication 1) $\Rightarrow 2$ ) follows from the vanishing of $H^{\bullet}\left(\mathbb{P}, \mathscr{O}_{\mathbb{P}}(-i)\right)$ for $1 \leq i \leq \operatorname{dim}(\mathbb{P})$. Assume that 2) holds. We will define by induction a sequence of 0 -regular sheaves $K_{i}$ on $\mathbb{P}$, for $0 \leq i \leq c$, such that:

a) $K_{0}=E$;

b) $K_{i+1}(-1)$ is the kernel of the evaluation map $H^{0}\left(\mathbb{P}, K_{i}\right) \otimes \mathscr{O}_{\mathbb{P}} \rightarrow K_{i}$; 
c) $H^{\bullet}\left(K_{i}(-j)\right)=0$ for $1 \leq j \leq n+i$.

Suppose the $K_{i}$ are defined for $0 \leq i \leq p$; we define $K_{p+1}$ by $b$ ). From the exact sequence $0 \rightarrow K_{p+1}(-1) \rightarrow H^{0}\left(\mathbb{P}, K_{p}\right) \otimes \mathscr{O}_{\mathbb{P}} \rightarrow K_{p} \rightarrow 0$ and $\left.c\right)$ we get $H^{\bullet}\left(K_{p+1}(-j)\right)=0$ for $1 \leq j \leq n+p+1$, and also $H^{q}\left(K_{p+1}(-q)\right)=H^{q-1}\left(K_{p}(-(q-1))\right)=0$. Thus $K_{p+1}$ is 0 -regular and satifies $\left.b\right)$ and $\left.c\right)$.

Put $L_{i}:=H^{0}\left(\mathbb{P}, K_{i}\right) \otimes \mathscr{O}_{\mathbb{P}}(-i)$; the exact sequences $0 \rightarrow K_{i+1}(-i-1) \rightarrow L_{i} \rightarrow K_{i}(-i) \rightarrow 0$ give a long exact sequence

$$
0 \rightarrow K_{c}(-c) \rightarrow L_{c-1} \rightarrow L_{c-1} \ldots L_{0} \rightarrow E \rightarrow 0 .
$$

Now $c$ ) means that $K_{c}$ is a Ulrich bundle on $\mathbb{P}$, hence trivial by the first part of the proof.

Remarks.-1) Condition 2) depends only on the line bundle $\mathscr{O}_{X}(1)$ (and not on the space of sections giving the embedding in $\mathbb{P})$. We will sometimes say that $E$ is a Ulrich bundle for $\left(X, \mathscr{O}_{X}(1)\right)$.

2) A direct sum of vector bundles is a Ulrich bundle iff each summand is a Ulrich bundle. Thus we need only to classify indecomposable Ulrich bundles.

Using the theorem, we can now answer our question on representation of hypersurfaces by linear determinants. Already in the case of quadrics, the result and the proof are far from trivial:

Proposition 3. Let $Q \subset \mathbb{P}^{n+1}$ be a smooth quadric. If $n$ is odd (resp. even) there is exactly one (resp. two) indecomposable Ulrich bundles on $Q$, of rank $2^{\left[\frac{n-1}{2}\right]}$.

As a consequence, we can write $\left(X_{1}^{2}+\ldots+X_{n}^{2}\right)^{r}$ as a determinant of linear forms if and only if $r$ is a multiple of $2^{\left[\frac{n-3}{2}\right]}$.

Proof : The projection $\pi: Q \rightarrow \mathbb{P}^{n}$ from a point $p \in \mathbb{P}^{n+1} \backslash X$ realizes $Q$ as a double covering of $\mathbb{P}^{n}$. We choose the coordinates so that $Q$ is given by an equation $X_{n+1}^{2}-q\left(X_{0}, \ldots, X_{n}\right)=0$, and $\pi\left(X_{0}, \ldots, X_{n+1}\right)=\left(X_{0}, \ldots, X_{n}\right)$. Thus $\pi$ is branched along the quadric $q=0$ in $\mathbb{P}^{n}$. This implies $\pi_{*} \mathscr{O}_{Q}=\mathscr{O}_{\mathbb{P}^{n}} \oplus \mathscr{O}_{\mathbb{P}^{n}}(-1)$, with the multiplication $\mathscr{O}_{\mathbb{P}^{n}}(-1) \otimes \mathscr{O}_{\mathbb{P}^{n}}(-1) \rightarrow \mathscr{O}_{\mathbb{P}^{n}}$ given by $q$.

The data of a vector bundle $E$ on $Q$ is equivalent to the data of the vector bundle $\pi_{*} E$ on $\mathbb{P}^{n}$, together with an algebra homomorphism $\varphi: \pi_{*} \mathscr{O}_{Q} \rightarrow \mathscr{E} n d\left(\pi_{*} E\right)$. If $E$ is a rank $r$ Ulrich bundle, we have $\pi_{*} E \cong \mathscr{O}_{\mathbb{P}^{n}}^{2 r}$, and $\varphi$ is given by a matrix $A \in \mathbf{M}_{2 r}(\mathbb{C}) \otimes_{\mathbb{C}} H^{0}\left(\mathbb{P}^{n}, \mathscr{O}_{\mathbb{P}^{n}}(1)\right)$, that is, a matrix $A=\left(A_{i j}(X)\right)$ whose entries are linear forms in $X=\left(X_{0}, \ldots, X_{n}\right)$, satisfying $A^{2}=q(X) \cdot I_{2 r}$.

Equivalently, we can view $A$ as a $\mathbb{C}$-linear map $\tilde{A}: \mathbb{C}^{n+1} \rightarrow \mathbf{M}_{2 r}(\mathbb{C})$, satisfying $\tilde{A}(v)^{2}=q(v) \cdot I_{2 r}$ for all $v$ in $\mathbb{C}^{n+1}$. This is the same as a $\mathbb{C}$-algebra homomorphism $C_{n+1} \rightarrow \mathbf{M}_{2 r}(\mathbb{C})$, where $C_{n+1}$ is the Clifford algebra $C\left(\mathbb{C}^{n+1}, q\right)$. If $n$ is odd, the algebra $C_{n+1}$ is simple, hence has a unique simple module, of dimension $2^{\frac{n+1}{2}}$, which corresponds to an indecomposable Ulrich bundle of rank $r=2^{\frac{n-1}{2}}$. If $n$ is even, $C_{n+1}$ is a product of two simple algebras of dimension $2^{n}$, hence has two simple modules of dimension $2^{\frac{n}{2}}$, corresponding to two non-isomorphic indecomposable Ulrich bundle of rank $2^{\frac{n-2}{2}}$.

Remark.- The bundles $\Sigma$ (resp. $\Sigma_{+}$and $\Sigma_{-}$) appear in $[\mathrm{K}]$, with a stronger result: the vector bundles $\left\langle\Sigma, \mathscr{O}_{Q}, \ldots, \mathscr{O}_{Q}(n-1)\right\rangle$ for $n$ odd, $\left\langle\Sigma_{-}, \Sigma_{+}, \mathscr{O}_{Q}, \ldots, \mathscr{O}_{Q}(n-1)\right\rangle$ for $n$ even form a semi-orthogonal decomposition of the (bounded) derived category of coherent sheaves on $Q$.

Using the notions of generalized Clifford algebra associated to a higher degree form and of matrix factorization, Herzog, Ulrich and Backelin have obtained a vast generalization of Proposition 3

Theorem 2 ([|[UB] $)$. Any smooth complete intersection $X \subset \mathbb{P}$ carries a Ulrich bundle. 


\section{SOME GENERAL PROPERTIES}

We first list a few direct consequences of Theorem 1 .

(2.1) Let $E$ be a rank $r$ Ulrich vector bundle on $X \subset \mathbb{P}$. Then $H^{i}(X, E(j))=0$ for all $j$ and $0<i<\operatorname{dim}(X)$ and $h^{0}(E)=r d$ (this follows from the condition 3) of the Theorem).

(2.2) A Ulrich bundle is semi-stable; if if it is not stable, it is an extension of Ulrich bundles of smaller rank (this follows again from condition 3).

(2.3) The Ulrich bundles on a curve $C \subset \mathbb{P}$ are the bundles $E(1)$, where $E$ is a vector bundle with vanishing cohomology.

(2.4) If $E$ is a Ulrich bundle on $X \subset \mathbb{P}$ and $Y$ is a hyperplane section of $X, E_{\mid Y}$ is a Ulrich bundle on $Y$. This follows from the exact sequence $0 \rightarrow E(-1) \rightarrow E \rightarrow E_{\mid Y} \rightarrow 0$.

(2.5) Let $E$ and $F$ be Ulrich bundles for $\left(X, \mathscr{O}_{X}(1)\right)$ and $\left(Y, \mathscr{O}_{Y}(1)\right)$; put $n:=\operatorname{dim}(X)$. Then $E \otimes F(n)$ is a Ulrich bundle for $\left(X \times Y, \mathscr{O}_{X}(1) \otimes \mathscr{O}_{Y}(1)\right)$.

Indeed we have $H^{\bullet}(X \times Y, E(-p) \otimes F(n-p))=H^{\bullet}(X, E(-p)) \otimes H^{\bullet}(Y, F(n-p))$. The first factor is zero for $1 \leq p \leq n$ and the second one for $n+1 \leq p \leq n+\operatorname{dim}(Y)$.

(2.6) Let $\pi: X \rightarrow Y$ be a finite surjective morphism, $L$ a very ample line bundle on $Y, E$ a vector bundle on $X$. Then $E$ is a Ulrich bundle for $\left(X, \pi^{*} L\right)$ if and only if $\pi_{*} E$ is a Ulrich bundle for $(Y, L)$.

This follows from the isomorphism $H \bullet\left(Y, \pi_{*} E \otimes L^{-k}\right) \cong H \bullet\left(X, E \otimes \pi^{*} L^{-k}\right)$.

Proposition 4. $\left(\mathbb{P}^{n}, \mathscr{O}_{\mathbb{P} n}(d)\right)$ admits a Ulrich bundle of rank $n !$.

Proof : Consider the quotient map $\pi:\left(\mathbb{P}^{1}\right)^{n} \rightarrow \operatorname{Sym}^{n} \mathbb{P}^{1}=\mathbb{P}^{n}$; the pull-back $\pi^{*} \mathscr{O}_{\mathbb{P}^{n}}(1)$ is $\mathscr{O}_{\mathbb{P}^{1}}(1) \otimes \ldots \otimes \mathscr{O}_{\mathbb{P}^{1}}(1)$. By (2.5) the line bundle $L=\mathscr{O}_{\mathbb{P}^{1}}(d-1) \otimes \ldots \otimes \mathscr{O}_{\mathbb{P}^{1}}(n d-1)$ is a Ulrich line bundle for $\left(\left(\mathbb{P}^{1}\right)^{n}, \pi^{*} \mathscr{O}_{\mathbb{P}^{n}}(d)\right)$, hence $\pi_{*} L$ is a Ulrich bundle for $\left(\mathbb{P}^{n}, \mathscr{O}_{\mathbb{P}^{n}}(d)\right)$ by (2.6).

In [ES] the authors use a much more sophisticated method to construct a homogeneous Ulrich

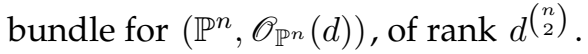

Corollary ([ES]). Let $X \subset \mathbb{P}$ be a smooth variety of dimension $n$, carrying a Ulrich bundle of rank $r$. Then $\left(X, \mathscr{O}_{X}(d)\right)$ carries a Ulrich bundle of rank $r n !$.

Proof : Let $E$ be a Ulrich bundle of rank $r$ for $X, \pi: X \rightarrow \mathbb{P}^{n}$ a finite projection, and $F$ a Ulrich bundle of rank $n$ ! for $\left(\mathbb{P}^{n}, \mathscr{O}_{\mathbb{P}^{n}}(d)\right)$. Then $E \otimes \pi^{*} F$ is a Ulrich bundle of rank $r n$ ! for $\left(X, \mathscr{O}_{X}(d)\right)$.

\section{ULRICH LINE BUNDLES}

Ulrich line bundles are rather exceptional; for instance, they cannot exist on a variety $X \subset \mathbb{P}$ with $\operatorname{Pic}(X)=\mathbb{Z} \mathscr{O}_{X}(1)$, except when $X$ is a linear subspace. Apart from the case of curves and projective spaces, there are a few situations where they do exist:

Proposition 5. 1) Let $S \subset \mathbb{P}$ be a del Pezzo surface (embedded by its anticanonical system). Let $L$ be a line bundle on $S$ with $L^{2}=-2, L \cdot K_{S}=0$. Then $L(1)$ is a Ulrich line bundle on $S$. We can take for instance $L=\mathscr{O}_{S}\left(\ell-\ell^{\prime}\right)$, where $\ell$ and $\ell^{\prime}$ are two disjoint lines.

2) Let $X \subset \mathbb{P}$ be a scroll over a curve $B$ : that is, there exists a fibration $p: X \rightarrow B$ whose fibers are linear subspaces in $\mathbb{P}$. Let $M$ be a line bundle on $B$ with $H \bullet(B, M)=0$. Then $p^{*} M \otimes \mathscr{O}_{X}(1)$ is a Ulrich line bundle on $X$. 
Proof : 1) We have $\chi(L)=0$ by Riemann-Roch, $H^{0}(S, L)=0$ since $L \cdot K_{S}=0$, and $H^{2}(S, L)=0$ since $\left(K_{S} \otimes L^{-1} \cdot K_{S}^{-1}\right)<0$, hence $H^{\bullet}(S, L)=0$. Then $H^{\bullet}\left(S, L^{-1}\right)=0$ for the same reason, hence $H^{\bullet}(S, L(-1))=0$ by Serre duality.

2) We have $R p_{*}\left(p^{*} M \otimes \mathscr{O}_{X}(-k)\right)=M \otimes R p_{*} \mathscr{O}_{X}(-k)$. The object $R p_{*} \mathscr{O}_{X}(-k)$ of $D(B)$ vanishes for $k=1, \ldots, \operatorname{dim}(X)-1$; for $k=0$ we get $H^{\bullet}\left(X, p^{*} M\right) \cong H \bullet(B, M)=0$.

Another interesting case is that of Enriques surfaces $S \subset \mathbb{P}[\overline{B N}]$. Suppose $S$ has no $(-2)$ curve. If we can express $\mathscr{O}_{S}(1)$ as $\mathscr{O}_{S}(A-B)$, where $A, B$ are divisors with $A^{2}=B^{2}=-2$, then $\mathscr{O}_{S}(A) \otimes \mathscr{O}_{S}(1)$ is a Ulrich line bundle, because $\mathscr{O}_{S}(A)$ and $\mathscr{O}_{S}(A) \otimes \mathscr{O}_{S}(-1) \cong \mathscr{O}_{S}(B)$ have vanishing cohomology. Borisov and Nuer conjecture that this is always possible, and prove it for the Fano model $S \subset \mathbb{P}^{5}$.

\section{RANK 2 ULRICH BUNDLES ON SURFACES}

Let $S \subset \mathbb{P}$ be a smooth projective surface. To find Ulrich bundles on $S$, a natural attack is to look for bundles $E$ such that $E(-1)$ and $E(-2)$ are Serre dual, so that the vanishing of $H^{\bullet}(E(-1))$ implies that of $H \bullet(E(-2))$. If $E$ has rank 2, this is achieved by imposing $\operatorname{det} E=K_{S}(3)$, which means that $E$ is special in the sense of [ES]: we say that a rank 2 Ulrich bundle $E$ on a smooth projective variety $X$ of dimension $n$ is special if $\operatorname{det} E=K_{X}(n+1)$. The existence of such a bundle implies that the Chow form of $X$ admits a "Bézout expression" as a Pfaffian (loc. cit.).

Proposition 6. Minimal surfaces of Kodaira dimension 0 admit a special rank 2 Ulrich bundle, except perhaps some special K3 surfaces.

Proof : Recall that there are four classes of such surfaces, namely K3, Enriques, abelian and bielliptic surfaces. The case of K3 surfaces is treated in [AFO], using the Lazarsfeld-Mukai construction. For each polarization type, the special surfaces for which the method does not apply form a strict subvariety of the moduli space.

We will give a uniform proof for the three remaining casest using the Serre construction in the following form (see for instance [HL, Theorem 5.1.1]). Let $S \subset \mathbb{P}$ be a smooth surface; using Remark $1(\S 1)$ we can assume that $S$ is embedded by the complete linear system $\left|\mathscr{O}_{S}(1)\right|$. Recall that a finite set of points $Z \subset S$ has the Cayley-Bacharach property if for every $p \in Z$, any hyperplane containing $Z \backslash\{p\}$ contains $Z$. If this is the case, there exists a rank 2 vector bundle $E$ and an extension

$$
0 \rightarrow K_{S} \rightarrow E \rightarrow \mathscr{I}_{Z}(1) \rightarrow 0 .
$$

Our aim is to show that a certain twist of $E(1)$ is a Ulrich bundle. We will use the following easy lemma :

Lemma 1. Let $S \subset \mathbb{P}$ be a smooth surface, and let $E$ be a rank 2 vector bundle on $S$ with $\operatorname{det} E=K_{S}(1)$, $h^{0}(E)=\chi(E)=0$. Then $E(1)$ is a special Ulrich bundle.

Proof: We have $K_{S} \otimes E^{*} \cong E(-1)$, hence $h^{2}(E)=h^{0}(E(-1))=0$. Since $\chi(E)=0$ this implies $H^{\bullet}(E)=0$, then $H^{\bullet}(E(-1))=H^{\bullet}\left(K_{S} \otimes E^{*}\right)=0$.

As a consequence, we have the following result, due to Casnati [C], and improving [B4]:

\footnotetext{
${ }^{1}$ The case of abelian surfaces was treated in [B3], and that of Enriques surfaces in [B] and [C].
} 
Proposition 7. Let $S \subset \mathbb{P}^{n}$ be a surface with $q=p_{g}=0$ and $H^{1}\left(S, \mathscr{O}_{S}(1)\right)=0$. Then $S$ admits a rank 2 special Ulrich bundle.

Proof : We choose for $Z$ a set of $n+2$ general points of $S$. They form a projective frame of $\mathbb{P}^{n}$, so $Z$ satisfies the Cayley-Bacharach property. We have $\chi\left(\mathscr{I}_{Z}(1)\right)=\chi\left(\mathscr{O}_{S}(1)\right)-n-2=-1$, hence $\chi(E)=0$. We have $H^{0}\left(\mathscr{I}_{Z}(1)\right)=0$ since $Z$ is general, hence $E(1)$ is a Ulrich bundle by Lemma 1 ,

Let us come back to Proposition 6 . Proposition7implies the case of Enriques surfaces. Let $S \subset \mathbb{P}^{n}$ be an abelian or bielliptic surface. We choose a smooth hyperplane section $C=S \cap H$ of $S$, and a subset of $n+1$ general points of $C$. Then any subset of $n$ points of $Z$ span $H$, hence $Z$ satisfies the Cayley-Bacharach property. Note that $g(C)=n+2$ by Riemann-Roch.

Let $\eta$ be an element of order 2 of $\operatorname{Pic}^{\circ}(S)$, with $\eta \neq \mathscr{O}_{S}, K_{S}$; we will apply Lemma 1 to $E \otimes \eta$. Since $\chi\left(\mathscr{O}_{S}\right)=0$ and $\chi\left(\mathscr{I}_{Z}(1)\right)=\chi\left(\mathscr{O}_{S}(1)\right)-n-1=0$, we have $\chi(E \otimes \eta)=\chi(E)=0$. Let us twist the exact sequence $(*)$ by $\eta$; we have $H^{\bullet}\left(K_{S} \otimes \eta\right)=H^{\bullet}(\eta)=0$, hence it suffices to prove $H^{0}\left(\mathscr{I}_{Z} \otimes \eta(1)\right)=0$. Since the restriction map $H^{0}(S, \eta(1)) \rightarrow H^{0}\left(C, \eta(1)_{\mid C}\right)$ is bijective, it suffices to show $H^{0}\left(C, \eta(1)_{\mid C}(-Z)\right)=0$. We have $\eta(1)_{\mid C}=K_{C} \otimes\left(\eta \otimes K_{S}^{-1}\right)_{\mid C} ;$ since $\eta \otimes K_{S}^{-1}$ is nontrivial, so is its restriction to $C$, hence $h^{0}\left(C, \eta(1)_{\mid C}\right)=g(C)-1=n+1$ and $h^{0}\left(C, \eta(1)_{\mid C}(-Z)\right)=0$ because $Z$ is general. Thus by Lemma1 $E \otimes \eta(1)$ is a special Ulrich bundle.

Remark. - It is quite possible that every rational surface carries a rank 2 Ulrich bundle. On the other hand, as observed in [ES, §6], a general surface $S \subset \mathbb{P}^{3}$ of degree $\geq 16$ does not carry a rank 2 Ulrich bundle. Indeed the existence of such a bundle on a surface $S$ with $\operatorname{Pic}(S)=\mathbb{Z}$ implies that the equation of $S$ can be written as a Pfaffian of linear forms [B1, Corollary 2.4], and this is not possible for a general $S \subset \mathbb{P}^{3}$ of degree $\geq 16$ [B1], Proposition 7.6].

\section{FANO THREEFOLDS OF INDEX 2}

Recall that a (smooth) Fano threefold $X$ has even index if there exists a line bundle $L$ on $X$ with $L^{2} \cong K_{X}^{-1}$. If $L$ is very ample, it embeds $X$ as a subvariety of degree $d$ of $\mathbb{P}^{d+1}$, with $d:=\left(L^{3}\right)$. There exist 7 families of such threefolds, with $3 \leq d \leq 8[\mathrm{II}]$; for $d=8$ we have $X=\mathbb{P}^{3}$ and $L=\mathscr{O}_{\mathbb{P}^{3}}(2)$, otherwise $X$ has index 2, that is, $L$ is not divisible in $\operatorname{Pic}(X)$.

Proposition 8. Every Fano threefold $X \subset \mathbb{P}^{d+1}$ of even index admits a special rank 2 Ulrich bundle.

Proof : Recall that a complete linear system of degree $n \geq 3$ on an elliptic curve $\Gamma$ defines an embedding $i: \Gamma \hookrightarrow \mathbb{P}^{n-1}$; the curve $i(\Gamma)$ is projectively normal, and is called a normal elliptic curve. We will prove in the Lemma below that the threefold $X$ contains a normal elliptic curve $\Gamma \subset \mathbb{P}^{d+1}$ (of degree $d+2$ ). Since $\omega_{\Gamma} \cong \mathscr{O}_{\Gamma}$, we have $\operatorname{det} N_{\Gamma / X} \cong \mathscr{O}_{\Gamma}(2)$. Since $H^{2}\left(X, \mathscr{O}_{X}(2)\right)=0$, there exists by the Serre construction (see e.g. [A]) a rank 2 vector bundle $E$ on $X$ and an exact sequence

$$
0 \rightarrow \mathscr{O}_{X} \rightarrow E \rightarrow \mathscr{I}_{\Gamma}(2) \rightarrow 0 \text {. }
$$

Let us show that $E$ is a Ulrich bundle. Since $\operatorname{det} E=\mathscr{O}_{X}(2)$, we have $E(-2) \cong K_{X} \otimes E(-2)^{*}$ and $E(-3) \cong K_{X} \otimes E(-1)^{*}$. Therefore using Serre duality it suffices to prove $H^{\bullet}(E(-1))=0$ and $H^{i}(E(-2))=0$ for $i=0,1$. But this follows from the equalities

$$
H^{\bullet}\left(\mathscr{O}_{X}(-1)\right)=H^{\bullet}\left(\mathscr{I}_{\Gamma}(1)\right)=0 \quad \text { and } \quad H^{i}\left(\mathscr{O}_{X}(-2)\right)=H^{i}\left(\mathscr{I}_{\Gamma}\right)=0 \text { for } i=0,1 .
$$


Lemma 2. $X$ contains a normal elliptic curve $\Gamma \subset \mathbb{P}^{d+1}$.

Proof : Assume first $d \leq 7$. Let $H$ be a general hyperplane in $\mathbb{P}^{d+1}$; the surface $S:=X \cap H$ is a Del Pezzo surface, obtained by blowing up $9-d$ points of $\mathbb{P}^{2}$ in general position. Consider the linear system of quartic curves in $\mathbb{P}^{2}$ passing doubly through two of these points, and simply through the others. It is easy to see that it is base point free, and therefore contains a smooth curve, which gives a normal elliptic curve $\Gamma_{0} \subset H$ of degree $d+1$.

Now let $p$ be a point of $\Gamma_{0}$, and let $L$ be a line contained in $X$ but not in $H$, and passing through $p$. We want to show that the curve $C=\Gamma_{0} \cup L$ can be deformed to a smooth curve $\Gamma$ in $X$, which must be a normal elliptic curve of degree $d+2$. According to [HH, Theorem 4.1], it suffices to prove $H^{1}\left(L, N_{L / X}\right)=0$ and $H^{1}\left(\Gamma_{0}, N_{\Gamma_{0} / X}(-p)\right)=0$. The normal bundle $N_{L / X}$ is equal to $\mathscr{O}_{L}^{2}$ or $\mathscr{O}_{L}(1) \oplus \mathscr{O}_{L}(-1)$ [IP, Lemma 3.3.4], hence its $H^{1}$ vanishes. The normal bundle $N_{\Gamma_{0} / X}$ is an extension of $\left(N_{S / X}\right)_{\mid \Gamma_{0}}=\mathscr{O}_{\Gamma_{0}}(1)$ by $N_{\Gamma_{0} / S}$; since both line bundles have degree $d+1 \geq 4$, we have $H^{1}\left(\Gamma_{0}, N_{\Gamma_{0} / X}(-p)\right)=0$, hence our assertion.

Now we consider the case $d=8$, so $X=\nu\left(\mathbb{P}^{3}\right) \subset \mathbb{P}^{9}$, where $\nu$ is the Veronese embedding given by the linear system $\left|\mathscr{O}_{\mathbb{P}^{3}}(2)\right|$. Let $\Gamma$ be a smooth elliptic curve of degree 5 in $\mathbb{P}^{3}$. We observe that a smooth quadric or a quadratic cone cannot contain an elliptic curve of degree 5 , so $\Gamma$ is not contained in a quadric; therefore the restriction map $\nu^{*}: H^{0}\left(\mathbb{P}^{3}, \mathscr{O}_{\mathbb{P}^{3}}(2)\right) \rightarrow H^{0}\left(\Gamma, \mathscr{O}_{\Gamma}(2)\right)$ is bijective, and $\nu(\Gamma)$ is a normal elliptic curve in $\mathbb{P}^{9}$.

Remarks.- 1) The existence of a Ulrich bundle (possibly of higher rank) could also be deduced case by case from the explicit description of these threefolds given in [II].

2) In the particular case of cubic threefolds, the Ulrich bundles given by the Proposition have already been constructed and studied in [MT]. They have a beautiful geometry, see [MT] and also [D] and [B2]. They are also considered for $d=4$ or 5 in [AC].

3) Conversely, any special Ulrich bundle $E$ of rank 2 on $X$ is of the above form. Indeed, let $s$ be a general section of $E$; the zero locus $\Gamma$ of $s$ is a smooth curve, and we have an exact sequence

$$
0 \rightarrow \mathscr{O}_{X} \stackrel{s}{\longrightarrow} E \rightarrow \mathscr{I}_{\Gamma}(2) \rightarrow 0 \text {. }
$$

Since $E$ is Ulrich, we have $h^{1}\left(\mathscr{I}_{\Gamma}\right)=0$ and $h^{2}\left(\mathscr{I}_{\Gamma}\right)=1$, hence $h^{0}\left(\mathscr{O}_{\Gamma}\right)=h^{1}\left(\mathscr{O}_{\Gamma}\right)=1$, so that $\Gamma$ is an elliptic curve. Moreover we have $h^{0}\left(\mathscr{I}_{\Gamma}(1)\right)=h^{1}\left(\mathscr{I}_{\Gamma}(1)\right)=0$, hence $\Gamma$ is a normal elliptic curve in $\mathbb{P}^{d+1}$.

4) If $3 \leq d \leq 5$, we have $\operatorname{Pic}(X)=\mathbb{Z}$, so any rank 2 Ulrich bundle is special and stable, because there are no Ulrich line bundles on $X$ (see (2.2)). It is easy to see that this is still the case for $d=7$ or 8 . However for $d=6$ there exist non-special rank 2 Ulrich bundles, and non-stable special rank 2 Ulrich bundles.

5) The same method produces a rank 2 Ulrich bundle on the Fano fourfolds of index $3 X \subset \mathbb{P}^{d+2}$ containing a del Pezzo surface of degree $d+2$. However these Fano fourfolds are not general. For instance, the cubic fourfolds containing a degree 5 del Pezzo surface are exactly the pfaffian cubics, that is, those which can be defined by the pfaffian of a $6 \times 6$ skew-symmetric matrix of linear forms; they form a hypersurface in the moduli space of cubic fourfolds [B1, Proposition 9.2].

We now consider the moduli space of rank 2 special Ulrich bundles on $X$; it is an open subset of the moduli space of semi-stable rank 2 bundles. 
Proposition 9. The moduli space $\mathscr{M}$ of rank 2 special Ulrich bundles on $X$ is smooth of dimension 5.

Proof : Let $\mathscr{H}$ be the Hilbert scheme of normal elliptic curves in $\mathbb{P}^{d+1}$ contained in $X$. Since $H^{1}\left(X, \mathscr{O}_{X}(2)\right)=0$, a curve $\Gamma$ in $\mathscr{H}$ determines the vector bundle $E$ in $\mathscr{M}$ up to isomorphism [A]. This defines a map $p: \mathscr{H} \rightarrow \mathscr{M}$ which is surjective (Remark 3 above); the fiber $p^{-1}(E)$ is canonically identified with an open subset of $\mathbb{P}\left(H^{0}(X, E)\right)$, so $p$ is smooth of relative dimension $2 d-1$.

Let $\Gamma \in \mathscr{H}$, and let $N$ be the normal bundle of $\Gamma$ in $X$. From the exact sequence $(*)$ we get an isomorphism $N \cong E_{\mid \Gamma}$. Therefore $\operatorname{deg} N=2 d+4$. We will prove that $H^{1}(\Gamma, N)=0$; this implies that the Hilbert scheme $\mathscr{H}$ is smooth of dimension $2 d+4$, hence that $\mathscr{M}$ is smooth of dimension 5 .

The Ulrich bundle $E$ admits a presentation

$$
\mathscr{O}_{\mathbb{P}}(-1)^{q} \stackrel{M}{\longrightarrow} \mathscr{O}_{\mathbb{P}}^{p} \rightarrow E \rightarrow 0
$$

where $M$ is a matrix of linear forms. Restricting to $\Gamma$, dualizing and taking cohomology, we get an exact sequence

$$
0 \rightarrow H^{0}\left(\Gamma, N^{*}\right) \rightarrow H^{0}\left(\Gamma, \mathscr{O}_{\Gamma}\right)^{p} \stackrel{{ }^{t} M}{\longrightarrow} H^{0}\left(\Gamma, \mathscr{O}_{\Gamma}(1)\right)^{q} .
$$

Let $v \in H^{0}\left(\Gamma, N^{*}\right)$; we have ${ }^{t} M v=0$ in $H^{0}\left(\Gamma, \mathscr{O}_{\Gamma}(1)\right)^{q} \cong H^{0}\left(\mathbb{P}^{d+1}, \mathscr{O}_{\mathbb{P}}(1)\right)^{q}$. Since $M$ is generically surjective, ${ }^{t} M$ is generically injective, so this implies $v=0$. Therefore $H^{0}\left(\Gamma, N^{*}\right)=0$, and $H^{1}(\Gamma, N)=0$ by Serre duality.

Examples.-1) Let $X$ be a cubic threefold, and let $J^{2} X$ be the translate of the intermediate Jacobian of $X$ which parametrizes 1-cycles of degree 2. The map $\mathscr{M} \rightarrow J^{2} X$ which associates to a rank 2 Ulrich bundle $E$ the class $c_{2}(E(-1))$ is an open embedding; its image is the complement of the divisor in $J^{2} X$ parametrizing sum of two lines [B2, Corollary 6.4].

2) Consider $X=\mathbb{P}^{3}$, embedded in $\mathbb{P}^{9}$ by the Veronese embedding. Any rank 2 Ulrich bundle on $X$ is the kernel of a homomorphism $T_{\mathbb{P}^{3}}(1) \rightarrow \mathscr{O}_{\mathbb{P}^{3}}(3)$ given by a contact form $\alpha \in H^{0}\left(\mathbb{P}^{3}, \Omega^{1}(2)\right)$ [ES, Proposition 5.11]. Thus the moduli space $\mathscr{M}$ is the open subset of contact forms in $\mathbb{P}\left(H^{0}\left(\mathbb{P}^{3}, \Omega^{1}(2)\right)\right)$, or equivalently the space of non-degenerate skew-symmetric forms on $\mathbb{C}^{4}$ up to a scalar.

Remark.- Assume $3 \leq d \leq 5$. Let $S$ be a very general surface in the linear system $\left|\mathscr{O}_{X}(2)\right|$, so that $S$ is a K3 surface with $\operatorname{Pic}(S)=\mathbb{Z}$. Let $\mathscr{M}_{S}$ be the moduli space of stable vector bundles on $S$ with $c_{1}=0, c_{2}=4$; it is an open subset of the holomorphic symplectic 10-dimensional manifold constructed by $\mathrm{O}^{\prime} \mathrm{Grady}[\mathrm{O}]$. The proof of [B2. Proposition 8.4] applies identically to show that the map $E \mapsto E(-1)_{\mid S}$ induces an isomorphism of $\mathscr{M}$ onto a Lagrangian subvariety of $\mathscr{M}_{S}$. However for $d=4$ or 5 these subvarieties do not seem to be part of a Lagrangian fibration.

\section{REFERENCES}

[A] E. Arrondo: A home-made Hartshorne-Serre correspondence. Rev. Mat. Complut. 20 (2007), no. 2, $423-443$.

[AC] E. Arrondo, L. Costa: Vector bundles on Fano 3-folds without intermediate cohomology. Comm. Algebra 28 (2000), no. 8, 3899-3911.

[AFO] M. Aprodu, G. Farkas, A. Ortega : Minimal resolutions, Chow forms and Ulrich bundles on K3 surfaces. Preprint arXiv:1212.6248. J. Reine Angew. Math., to appear.

[B1] A. Beauville: Determinantal hypersurfaces. Mich. Math. J. 48 (2000), 39-64.

[B2] A. Beauville: Vector bundles on the cubic threefold. Symposium in Honor of C. H. Clemens, 71-86, Contemp. Math. 312, Amer. Math. Soc., Providence, RI, 2002. 
[B3] A. Beauville: Ulrich bundles on abelian surfaces. Proc. Amer. Math. Soc. 144 no. 11 (2016), 4609-4611.

[B4] A. Beauville: Ulrich bundles on surfaces with $p_{g}=q=0$. Preprint arXiv:1607.00895.

[BN] L. Borisov, H. Nuer: Ulrich bundles on Enriques surfaces. Preprint arXiv:1606.01459.

[C] G. Casnati: Special Ulrich bundles on non-special surfaces with $p_{g}=q=0$. Preprint arXiv:1609.07915.

[D] S. Druel: Espace des modules des faisceaux de rang 2 semi-stables de classes de Chern $c_{1}=0, c_{2}=2$ et $c_{3}=0$ sur la cubique de $\mathbb{P}^{4}$. Internat. Math. Res. Notices 2000, no. 19, 985-1004.

[ES] D. Eisenbud, F.-O. Schreyer: Resultants and Chow forms via exterior syzygies. J. Amer. Math. Soc. 16 (2003), no. 3, 537-579.

[HH] R. Hartshorne, A. Hirschowitz: Smoothing algebraic space curves. Algebraic geometry, Sitges, 98-131; Lecture Notes in Math. 1124, Springer, Berlin (1985).

[HL] D. Huybrechts, M. Lehn: The geometry of moduli spaces of sheaves (2nd edition). Cambridge University Press, Cambridge, 2010.

[HUB] J. Herzog, B. Ulrich, J. Backelin : Linear maximal Cohen-Macaulay modules over strict complete intersections. J. Pure Appl. Algebra 71 (1991), no. 2-3, 187-202.

[IP] V.A. Iskovskikh, Y. Prokhorov: Fano varieties. Algebraic geometry V; Encycl. Math. Sci. 47, Springer, Berlin (1999).

[K] M. Kapranov: On the derived categories of coherent sheaves on some homogeneous spaces. Invent. Math. 92 (1988), no. 3, 479-508.

[M] D. Mumford: Lectures on curves on an algebraic surface. Annals of Mathematics Studies 59, Princeton University Press (1966).

[MT] D. Markushevich, A. Tikhomirov: The Abel-Jacobi map of a moduli component of vector bundles on the cubic threefold. J. Algebraic Geom. 10 (2001), no. 1, 37-62.

[O] K. O' Grady: Desingularized moduli spaces of sheaves on a K3. J. Reine Angew. Math. 512 (1999), 49-117.

[S] H.E. Schröter : Nachweis der 27 Geraden auf der allgemeinen Oberflche dritter Ordnung. J. Reine Angew. Math. 62 (1863), 265-280.

[U] B. Ulrich: Gorenstein rings and modules with high numbers of generators. Math. Z. 188 (1984), no. 1, 23-32.

Université Côte d'Azur, CNRS - Laboratoire J.-A. Dieudonné, ParC VAlrose, F-06108 Nice Cedex 2, France

E-mail address: arnaud.beauville@unice.fr 